\title{
МНОГОКРИТЕРИАЛЬНАЯ ОЦЕНКА ЭФФЕКТИВНОСТИ ГРУППОВОГО ПОЛЕТА ЛЕТАТЕЛЬНЫХ АППАРАТОВ С ПОМОЩЬЮ МУЛЬТИПЛИКАТИВНОЙ ФОРМЫ
}

\author{
() 2020 Г. Н. Лебедев ${ }^{1}$, Д. А. Михайлин ${ }^{1}$, О. Ю. Царева ${ }^{\varpi 1}$, М. Е. Чернякова ${ }^{2}$ \\ ${ }^{1}$ Московский авиационный институт (национальный исследовательский университет) \\ Волоколамское шоссе, 4, 125993 Москва, Российская Федерация \\ ${ }^{2}$ Московский филиал по беспилотному направлению, ООО «Группа-Кронштадт» \\ Андропова пр-т,18, 115432 Москва, Российская Федерация
}

\begin{abstract}
Аннотация. Рассматривается многокритериальная задача оценки эффективности группового полета пилотируемой и беспилотной авиации при обслуживании потока заявок. С помощью динамического программирования сформирована модель назначения динамических приоритетов, оценивающих важность обслуженных заявок. Осуществлен выбор единого критерия оценки эффективности в мультипликативной форме, в которой количество сомножителей равно числу учитываемых факторов. Компьютерное моделирование показало преимущество в эффективности планирования с помощью мультипликативной формы по сравнению с аддитивной за счет повышения суммарной важности обслуженных объектов в маршрутном полете.

Ключевые слова: обслуживание заявок, динамическое программирование, компьютерное моделирование, критерий оценки эффективности.
\end{abstract}

\section{ВВЕДЕНИЕ}

В данной работе решается многокритериальная задача оценки эффективности группового полета пилотируемой и беспилотной авиации при обслуживании потока заявок. Для пилотируемой авиации под заявкой понимается требование прилететь в исходную точку пункта с последующим перелетом в заданную конечную точку. Для беспилотной авиации понимается требование обеспечить наблюдение очередного наземного пункта при пролете над ним одного из беспилотных летательных аппаратов (БЛА).

Существует ряд практически важных многокритериальных задач оценки эффективности систем, в том числе задач их оптимизации [1-8], когда необходимо учесть одни параметры в виде ограничений, а другие - в виде

Царева Ольга Юрьевна e-mail: olyatsareva@yandex.ru показателей улучшаемого качества. К ним, в частности, относится задача оптимизации систем обучения [8-13], а также задача параметрической оптимизации технических систем управления летательными аппаратами [14-16].

К числу локальных критериев эффективности можно отнести, в том числе суммарную важность полученных результатов обслуживания заявок, экономичность группового полета и своевременность обслуживания с учетом возможного пребывания заявок в очереди. Сложность решаемой задачи состоит в комплексировании влияния нескольких факторов в форме единого критерия эффективности.

Анализ результатов предшествующих работ показал, что в качестве основного инструмента единой оценки эффективности решения многокритериальных задач используется аддитивный критерий. Прежде всего, к ним относится задача линейного програмКонтент доступен под лицензией Creative Commons Attribution 4.0 License. The content is available under Creative Commons Attribution 4.0 License. 


\section{Г. Н. Лебедев, Д. А. Михайлин, О. Ю. Царева, М. Е. Чернякова}

мирования, а также более близкая к данной работе задача целочисленного программирования при поиске маршрута полета в классической «задаче о коммивояжере» - метод ветвей и границ. В любой из этих задач минимизируется сумма штрафных функций, выбираемых на каждом шаге оптимизации.

Более того, в задачах оптимизации группового полета его общая эффективность оценивается аддитивной суммой действий каждого летательного аппарата в отдельности. Аналогичный подход к оценке групповых действий наблюдается в технических задачах операторного управления сложными объектами, системах обучения групповым навыкам, при оценке эффективности деятельности вузов и т. д.

Их общий недостаток состоит в отсутствии учета влияния поведения друг на друга представителей группы. Также не уделяется внимание и тому, что ряд факторов в динамической обстановке является переменной величиной, и эти изменения тоже необходимо учесть.

В связи с этим ставится задача формирования такого единого критерия оценки эффективности, который, с одной стороны, учел бы в своей свертке взаимовлияние различных частных показателей, имеющих в том числе неодинаковую физическую размерность, и, с другой стороны, в количественной форме оценил переменную важность наземных объектов из-за задержек их обслуживания, изменяемую удаленность этих объектов от группы летательных аппаратов и растущую несвоевременность обслуживания объектов согласно требуемому графику.

Для решения поставленной задачи требуется:

- сформировать математическую модель назначения динамических приоритетов переменной важности первоочередных объектов на каждом шаге планирования группового полета;

- обосновать единую форму критерия эффективности группового полета с учетом взаимосвязи частных показателей качества;

- сравнить предложенную оценку эффективности с известным подходом с помощью компьютерного моделирования.

\section{1. МАТЕРИАЛЫ И МЕТОДЫ ИССЛЕДОВАНИЯ}

\section{1. Формирование модели назначения приоритетов, оценивающих важность обслуженных заявок, с помощью динамического программирования}

При оценке степени важности обсуживаемой заявки следует различать априорную важность, назначенную экспертным путем, и апостериорную важность, учитывающую динамику нарастания этой важности с увеличением времени ожидания в очереди обслуживаемой заявки. В данной работе априорные важности представляются в виде двух значений: считается, что обслуживание указанной выше в постановке задачи для мобильных объектов (MO) имеет наибольшую важность $B_{j}$, лежащую в заданных пределах:

$$
B_{\min } \leq B_{j} \leq B_{\text {max }} \text {, }
$$

а остальные типы неподвижных пунктов или объектов-ориентиров имеют существенно меньшую важность $b_{j}$ :

$$
b_{\text {min }} \leq b_{m} \leq b_{\max } ; b_{\max } \leq 0,5 B_{\max }, b_{\text {min }} \leq 0,5 B_{\text {min }} \text {. }
$$

Объединяя вышесказанное, можно записать:

для $\mathrm{MO}$ :

$\dot{B}_{j}=\left\{\begin{array}{c}\left(B_{\max }-B_{j}\right) \alpha_{j} \rightarrow \text { вне обслуживания } \\ -\frac{B_{j} P_{j}}{\Delta t_{j}} \rightarrow \text { в конце каждого } \\ \text { обслуживания; }\end{array}\right.$

для остальных неподвижных объектов:

$\dot{b}_{m}=\left\{\begin{array}{l}\left(b_{\max }-b_{m}\right) \boldsymbol{\alpha}_{m} \rightarrow \text { вне обслуживания } \\ -\frac{b_{m}}{\Delta \tau} \rightarrow \text { в конце обслуживания }\end{array}\right.$

В свою очередь динамика нарастания важности прежде всего зависит от доминирующего параметра качества обслуживания, которым является время $\tau_{i}$ «необслуживания» $j$-го пункта, растущее между соседними по времени контактами с БЛА, и обнуляющееся сразу после обслуживания, так как интерес к $j$-му объекту в этой ситуации пропадает:

$$
\dot{\tau}_{i}=\left\{\begin{array}{l}
1, \text { при } i \neq j \\
-\frac{\tau_{i}}{\Delta t}, \text { при } i=j
\end{array} .\right.
$$


Многокритериальная оценка эффективности группового полета летательных аппаратов...

Кроме того считается, что между параметрами динамической важности и временем $\tau_{i}$ задержки в обслуживании каждого пункта нет никакой статистической зависимости. При этих допущениях вычислялись соответствующие динамические приоритеты для различных конкретных практических задач.

В частности, в данной работе решена конкретная задача назначения динамических приоритетов для обслуживания пунктов, когда математическая модель роста важности описывается не в виде дифференциального уравнения первого порядка, а в виде динамической системы второго порядка:

$$
\begin{aligned}
& \dot{x}_{1 i}=\left\{\begin{array}{l}
x_{2 j}, \text { при } i \neq j \\
-\frac{x_{1 j}}{\Delta t}, \text { при } i=j
\end{array}\right. \\
& \dot{x}_{2 i}=\left\{\begin{array}{l}
a_{i}, \text { при } i \neq j \\
-\frac{x_{2 j}}{\Delta t}, \text { при } i=j
\end{array}\right.
\end{aligned}
$$

где $a_{i}$ - ускорение роста важности; $x_{2 i}-$ скорость нарастания важности каждого из обслуживаемых пунктов; $x_{1 i}$ - текущее значение важности $j$-го пункта; $j-$ номер обслуживаемого в текущий момент пункта.

Решение задачи было получено с помощью метода динамического программирования [17]. Согласно этому методу необходимо использовать уравнение Беллмана в частных производных, которое имеет вид:

$$
-\frac{\partial \boldsymbol{\varepsilon}}{\partial t}=\min _{j}\left\{\begin{array}{c}
\sum_{i=1}^{n} d_{i} x_{1 i} \tau_{i}+ \\
\left.+\sum_{i \neq j}^{n}\left(\frac{\partial \boldsymbol{\varepsilon}}{\partial x_{1 i}} x_{2 i}+\frac{\partial \boldsymbol{\varepsilon}}{\partial x_{2 i}} a_{i}+\frac{\partial \boldsymbol{\varepsilon}}{\partial \tau_{i}}\right)-\right\}_{(6)}^{(6)} \\
-\left(\frac{\partial \boldsymbol{\varepsilon}}{\partial x_{1 j}} \frac{x_{1 j}}{\Delta t}+\frac{\partial \boldsymbol{\varepsilon}}{\partial x_{2 j}} \frac{x_{2 j}}{\Delta t}+\frac{\partial \boldsymbol{\varepsilon}}{\partial \boldsymbol{\tau}_{j}} \frac{\boldsymbol{\tau}_{j}}{\Delta t}\right)
\end{array}\right)
$$

где $\boldsymbol{\varepsilon}\left(\bar{x}_{1}, \bar{x}_{2}, \bar{\tau}\right)-$ искомая функция Беллмана при оптимальном поведении.

Также как и АКОР, решить это уравнение можно с помощью представления функции Беллмана $\boldsymbol{\varepsilon}$ в виде степенного полинома второго порядка с неизвестными коэффициентами, которые необходимо определить:

$$
\begin{gathered}
\boldsymbol{\varepsilon}=\sum_{i=1}^{n}\left[\left(\boldsymbol{\beta}_{1 i} x_{1 i}+\boldsymbol{\gamma}_{1 i} \frac{x_{1 i}^{2}}{2}\right)+\left(\boldsymbol{\beta}_{2 i} x_{2 i}+\boldsymbol{\gamma}_{2 i} \frac{x_{2 i}^{2}}{2}\right)+\right. \\
+\left(\boldsymbol{\beta}_{3 i} \boldsymbol{\tau}_{i}+\boldsymbol{\gamma}_{3 i} \frac{\boldsymbol{\tau}_{i}^{2}}{2}\right)+ \\
+\left(\boldsymbol{\psi}_{12 i} x_{1 i} x_{2 i}+\boldsymbol{\psi}_{13 i} x_{1 i} \boldsymbol{\tau}_{i}+\boldsymbol{\psi}_{23 i} x_{2 i} \boldsymbol{\tau}_{i}\right),
\end{gathered}
$$

где $\boldsymbol{\beta}_{1 i}, \boldsymbol{\beta}_{2 i}, \boldsymbol{\beta}_{3 i}, \boldsymbol{\gamma}_{1 i}, \boldsymbol{\gamma}_{2 i}, \boldsymbol{\gamma}_{3 i}, \boldsymbol{\psi}_{12 i}, \boldsymbol{\psi}_{13 i}, \boldsymbol{\psi}_{23 i}$-искомые коэффициенты.

Нахождение этих коэффициентов возможно с помощью метода рабочей точки, согласно которого в окрестности этой точки вычисляются ординаты $C_{i}$ минимального риска, которые необходимо приравнять друг к другу.

В настоящей работе согласно (7) искомыми являются девять коэффициентов $\boldsymbol{\beta}_{j}, \boldsymbol{\gamma}_{j}$, $\boldsymbol{\psi}_{j k}$. Поэтому зададимся следующими десятью ординатами риска, чтобы, приравняв их друг к другу, получить систему необходимых линейных алгебраических уравнений:

$$
\begin{gathered}
4 \Delta x_{1} \boldsymbol{\psi}_{12 j}+\boldsymbol{\beta}_{2 j}+3 \Delta x_{2} \gamma_{2 j}+2 \Delta \tau \psi_{23 j}=0, \\
\boldsymbol{\beta}_{1 j}+\gamma_{1 j} 3 \Delta x_{1}+4 \Delta \tau \psi_{13 j}+2 \Delta x_{2} \boldsymbol{\psi}_{12 j}=2 d_{j} \Delta \tau \Delta t, \\
\boldsymbol{\beta}_{3 j}+3 \Delta \tau \gamma_{3 j}+4 \Delta x_{2} \boldsymbol{\Psi}_{23 j}+2 \Delta x_{1} \boldsymbol{\psi}_{13 j}=d_{j} \Delta x_{1} \Delta \tau, \\
{\left[\boldsymbol{\beta}_{1 j}+3 \Delta x_{1} \boldsymbol{\gamma}_{1 j}+2 \Delta \tau \boldsymbol{\psi}_{13 j}\right] \Delta x_{1}-} \\
-\left[\boldsymbol{\beta}_{2 j}+3 \Delta x_{2} \boldsymbol{\gamma}_{2 j}+2 \Delta \tau \boldsymbol{\psi}_{23 j}\right] \Delta x_{2}=d_{j} \Delta x_{1} \Delta \tau \\
{\left[\boldsymbol{\beta}_{1 j}+3 \Delta x_{1} \gamma_{1 j}+2 \boldsymbol{\psi}_{12 j} \Delta x_{2}\right] \Delta x_{1}-} \\
-\left[\boldsymbol{\beta}_{3 j}+3 \Delta \tau \gamma_{3 j}+2 \Delta x_{2} \boldsymbol{\Psi}_{23 j}\right] \Delta \tau=0
\end{gathered}
$$

$\boldsymbol{\beta}_{1 j}+3 \Delta x_{1} \Delta x_{2} \gamma_{1 j}+2 \psi_{12 j} \Delta x_{2}+2 \psi_{13 j} \Delta \tau=d_{j} \Delta x_{1} \Delta \tau$.

Для получения окончательного результата подставим в формулу приоритета найденные значения коэффициентов функции Беллмана и, пренебрегая рядом членов из-за их малости, упростим это выражение

$$
\Pi_{j} \approx d_{j}\left(x_{1 j}+\Delta t x_{2 j}\right)\left(\tau_{j}+\frac{1,5 \Delta \tau^{2}}{\Delta t}\right) .
$$

В эту формулу нужно добавить еще один сомножитель, учитывающий своевременность обслуживания пунктов при взаимодействии с БЛА. Поэтому можно записать

$$
\Pi_{j}=a_{j} d_{j}\left(\tau_{j}+\Delta t\right)\left(\frac{\tau_{j}}{\tau_{\max }}+m_{1}\right)\left(\frac{\Delta t_{j}}{\Delta t_{\max }}+m_{2}\right) \text {. }
$$

Видно, что в данной конкретной задаче динамический приоритет П пликативную форму. 


\section{Г. Н. Лебедев, Д. А. Михайлин, О. Ю. Царева, М. Е. Чернякова}

\section{2. Выбор единого критерия оценки эффективности в мультипликативной форме}

При решении различных многокритериальных задач обычной является аддитивная модель [18], когда критерием общей оценки является среднее значение отдельных частных показателей $x_{i}$ или их общая сумма в баллах

$$
J=\max \sum_{i=1}^{n} x_{i}
$$

где $x_{i}$ - показатели, требующие учета.

Однако в ряде случаев эта оценка не учитывает, как различную важность отдельных показателей, так и их возможную несбалансированность. Например, в системах обучения [19] одну и ту же сумму баллов пяти показателей имеют два обучаемых лица - 3, 3, $3,3,4$ и 2, 2, 3, 4, 5, в то время как первое обучаемое лицо явно предпочтительнее второго (которого обычно отчисляют из института). Между тем произведение этих же показателей у второго лица явно меньше, чем у первого. Поэтому представление максимизируемого критерия в виде взвешенной суммы аддитивной и мультипликативной форм показателей является более целесообразной сверткой

$$
J=\max \left(c_{1} \sum_{i=1}^{n} x_{i}+c_{1} \prod_{i=1}^{n} x_{i}\right) .
$$

Использование свертки (12) в качестве критерия оптимизации позволяет исключить «узкие места» в системе, когда один из показателей $x_{i} \rightarrow 0$, поскольку в этом случае второе слагаемое в формуле (12) сразу же обнуляется.

Применительно к рассматриваемой в настоящей работе задаче необходимо, во-первых, все три максимизируемых показателя превратить в безразмерные путем их деления на максимальные значения

$$
\begin{gathered}
x_{1}=\frac{r_{\text {min }}}{r} ; x_{2}=\frac{\Delta t_{\text {min }}}{\Delta t} ; \\
x_{3}=\frac{B}{B_{\max }},
\end{gathered}
$$

где $r_{\min }-$ минимальная общая длина маршрута; $\Delta t_{\min }-$ минимальное общее время несоблюдения графика; $B_{\max }-$ максимально возможная суммарная важность пунктов, попавших в маршрут.

Во-вторых, учтем фактор неодинаковой значимости каждого нормированного показателя $x_{i}$ с помощью весовых коэффициентов $c_{1}, c_{2}, c_{3}$, назначаемых потребителем. В-третьих, представим свертку $J$ еще более компактно в виде

$$
J_{0}=\max \left(x_{1}+m_{1}\right)\left(x_{2}+m_{2}\right)\left(x_{3}+m_{3}\right) \text {, }
$$

где $m_{1}=\sqrt{\frac{c_{2} c_{3}}{c_{1}}} ; m_{2}=\sqrt{\frac{c_{1} c_{3}}{c_{2}}} ; m_{3}=\sqrt{\frac{c_{1} c_{2}}{c_{3}}}$.

При этом $\sum_{i=1}^{3} m_{i}=1$.

Тогда свертка $J_{0}$ более подробно состоит из членов степенного ряда:

$$
\begin{aligned}
& J_{0}=\max \left(\begin{array}{l}
x_{1} m_{2} m_{3}+x_{2} m_{1} m_{3}+ \\
+x_{3} m_{1} m_{2}+x_{1} x_{2} m_{3}+ \\
+x_{1} x_{3} m_{2}+x_{2} m_{3} m_{1}+x_{1} x_{2} x_{3}
\end{array}\right)= \\
& =\max \left(\begin{array}{l}
c_{1} x_{1}+c_{2} x_{2}+c_{3} x_{3}+x_{1} x_{2} \sqrt{\frac{c_{1} c_{2}}{c_{3}}}+ \\
+x_{1} x_{3} \sqrt{\frac{c_{1} c_{3}}{c_{2}}}+x_{2} x_{3} \sqrt{\frac{c_{2} c_{3}}{c_{1}}}+x_{1} x_{2} x_{3}
\end{array}\right) .
\end{aligned}
$$

Достоинство предлагаемой нелинейной свертки состоит в том, что для нее достаточно задать всего три числа $c_{1}, c_{2}, c_{3}$. Это доказывает возможность ее использования в более общих случаях, если учесть фактор неодинаковой значимости каждого нормированного показателя $x_{i}$ с помощью весовых коэффициентов $m_{1}, m_{2}, m_{3}$ назначаемых потребителем. Тогда можно представить приоритет $\Pi$ в наиболее компактной форме $[20,21]$

$$
\Pi=\max \left(x_{1}+m_{1}\right)\left(x_{2}+m_{2}\right)\left(x_{3}+m_{3}\right) .
$$

Достоинство предлагаемой нелинейной свертки состоит в том, что для нее достаточно задать всего три числа $m_{1}, m_{2}, m_{3}$ Можно также пойти дальше в обобщении формулы (16), если учитывать ненулевую скорость движения обслуживаемых наземных пунктов, тогда в формулу (16) можно добавить еще один множитель $\frac{V_{\text {ц }}}{V_{0}+V_{\text {ц }}}$, который растет пропорционально скорости $V_{\text {ц }}$ (то есть с увеличением скорости движения обслуживаемого наземного объекта, его приоритет растет). Поэ- 
Многокритериальная оценка эффективности группового полета летательных аппаратов...

тому эвристическое правило (16) должно быть дополнительно четвертым сомножителем

$$
\begin{aligned}
J= & \max _{j}\left(\frac{b_{j}}{b_{\max }}+m_{1}\right)\left(\frac{r_{\min }}{\min _{j} r_{i j}}+m_{2}\right) \times \\
& \times\left(\frac{\Delta t_{\min }}{\Delta t_{j}}+m_{3}\right)\left(\frac{V_{j}}{V_{0}}+m_{4}\right) .
\end{aligned}
$$

Если, кроме того, учитывать при назначении динамических приоритетов труднодоступность достижения каждого наземного объекта при подлете к нему, то критерий (17) должен быть дополнен пятым сомножителем, если эта доступность неодинакова:

$$
\begin{gathered}
J=\max _{j}\left(\frac{b_{j}}{b_{\max }}+m_{1}\right)\left(\frac{r_{\min }}{\min _{j} r_{i j}}+m_{2}\right) \times \\
\times\left(\frac{\Delta t_{\min }}{\Delta t_{j}}+m_{3}\right)\left(\frac{V_{j}}{V_{0}}+m_{4}\right)\left(\frac{P_{\min }}{P_{j}+P_{\min }}+m_{5}\right),
\end{gathered}
$$

где $P_{\min }-$ минимальная вероятность потери работоспособности ЛА, а $P_{j}-$ вероятность потери работоспособности ЛА при подлете к обслуживаемому объекту.

Таким образом, на основании динамического программирования найдена компактная мультипликативная форма критерия назначения динамических приоритетов для выбора, которая эффективнее аддитивного за счет исключения «узких мест» в формируемом плане полета.

\section{2. РЕЗУЛЬТАТЫ ИССЛЕДОВАНИЙ И ИХ ОБСУЖДЕНИЕ}

В качестве исходных данных при моделировании сравниваемых критериев приняты следующие допущения.

1. Задано общее число $n$ наземных объектов (как неподвижных точечных или размерных, так и трасс движения МО).

2. Среди них задано число $M$ объектов, для которых указан график их доступного наблюдения с помощью параметра $t_{j}-$ это наиболее благоприятный момент наблюдения по времени.

3. Заданы три координаты для каждого пункта: $X_{m} ; Z_{m} ; Y_{m}$ для наблюдения МО; $m=1, \ldots M$, где $Y_{m}$ - требуемая высота наблю- дения, а также координаты «обычных» пунктов, доступных для наблюдения в любое время $-X_{i} ; Z_{i} ; Y_{i}$.

4. Известны априорные важности $B_{m}, b_{i}$ наблюдаемых объектов при условии, что $B_{m} \gg b_{i}$, т. е. наблюдение МО по графику важнее остальных в данном вылете БЛА (например, $\left.B_{m}=10, b_{i}=1, \ldots 6\right)$.

5. Заданы координаты начальной и конечной точек $A$ и $B$ маршрута.

6. Требуемый график обслуживания МO представлен в табл. 1.

Таблица 1. Грабик обслуживания мобильных объектов

[Table 1. Mobile objects service schedule]

\begin{tabular}{|c|c|c|c|c|c|c|c|}
\hline$j$ & 1 & 2 & 3 & 4 & 5 & 6 & 7 \\
\hline$i$ & 6 & 13 & 19 & 21 & 25 & 33 & 40 \\
\hline$t_{j}$ & 150 & 300 & 450 & 520 & 640 & 790 & 900 \\
\hline$\tau$ & 10 & 10 & 10 & 10 & 10 & 10 & 10 \\
\hline
\end{tabular}

7. Конец полета согласно регламенту имеет заданное время $t_{k}=900$ мин, а скорость БЛА: $V_{\text {БЛА }}=144 \kappa \mathcal{\kappa M} / \boldsymbol{u}=40 \mathrm{M} / \mathrm{c}$.

8. Требуется сформировать маршрут облета пунктов (может быть не всех) при условии, что в одном пункте нужно быть один раз.

Исходные данные для моделирования представлены на рис. 1, где 6, 13, 19, 21, 25, $33,40 \rightarrow$ MO.

Конкретные численные данные местоположения объектов приведены в табл. 2 .

Таблица 2. Данные обо всех объектах, включая мобильные объекты

[Table 2. Data on all facilities, including mobile facilities]

\begin{tabular}{|c|c|c|c|c|c|}
\hline$i$ & $\begin{array}{c}\text { object } \\
\text { type }\end{array}$ & $x$ & $y$ & $b$ & $t$ \\
\hline 0 & mobile & 0 & 0 & 0 & 0 \\
\hline 1 & simple & 40000 & 168000 & 3 & \\
\hline 2 & lkks & 100000 & 108000 & 6 & \\
\hline 3 & simple & 130000 & -116000 & 3 & \\
\hline 4 & simple & 280000 & 238000 & 3 & \\
\hline 5 & lkks & 360000 & -265000 & 6 & \\
\hline 6 & mobile & 440000 & -67000 & 10 & 15000 \\
\hline 7 & simple & 490000 & 25000 & 3 & \\
\hline 8 & lkks & 570000 & 155000 & 6 & \\
\hline
\end{tabular}




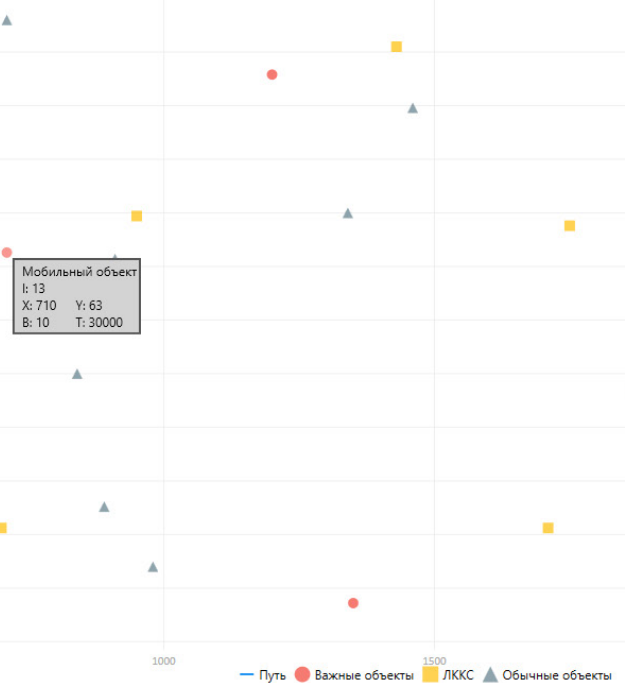

Рис. 1. Исходные данные расположения объектов наблюдения при компьютерном моделировании [Fig.1. Initial data on the location of observation objects during computer modeling]

\begin{tabular}{|c|c|c|c|c|c|}
\hline 9 & lkks & 640000 & -37000 & 6 & \\
\hline 10 & simple & 670000 & 166000 & 3 & \\
\hline 11 & lkks & 700000 & -194000 & 6 & \\
\hline 12 & simple & 710000 & 280000 & 3 & \\
\hline 13 & mobile & 710000 & 63000 & 10 & 30000 \\
\hline 14 & simple & 840000 & -50000 & 3 & \\
\hline 15 & simple & 890000 & -174000 & 3 & \\
\hline 16 & simple & 910000 & 57000 & 3 & \\
\hline 17 & lkks & 950000 & 97000 & 6 & \\
\hline 18 & simple & 980000 & -230000 & 3 & \\
\hline 19 & mobile & 1200000 & 229000 & 10 & 45000 \\
\hline 20 & simple & 1340000 & 100000 & 3 & \\
\hline 21 & mobile & 1350000 & -264000 & 10 & 52000 \\
\hline 22 & lkks & 1430000 & 255000 & 6 & \\
\hline 23 & simple & 1460000 & 198000 & 3 & \\
\hline 24 & lkks & 1710000 & -194000 & 6 & \\
\hline 25 & mobile & 1870000 & -66000 & 10 & 64000 \\
\hline 26 & lkks & 1750000 & 88000 & 6 & \\
\hline 27 & simple & 2120000 & -62000 & 3 & \\
\hline 28 & simple & 2290000 & 165000 & 3 & \\
\hline 29 & simple & 2330000 & 20000 & 3 & \\
\hline 30 & lkks & 2390000 & 239000 & 6 & \\
\hline 31 & lkks & 2420000 & -193000 & 6 & \\
\hline 32 & simple & 2440000 & 79000 & 3 & \\
\hline 33 & mobile & 2460000 & -19000 & 10 & 79000 \\
\hline
\end{tabular}

\begin{tabular}{|l|c|c|c|c|c|}
\hline 34 & simple & 2490000 & 282000 & 3 & \\
\hline 35 & lkks & 2550000 & -228000 & 6 & \\
\hline 36 & simple & 2710000 & -154000 & 3 & \\
\hline 37 & lkks & 2760000 & 80000 & 6 & \\
\hline 38 & simple & 2850000 & 219000 & 3 & \\
\hline 39 & simple & 2890000 & 156000 & 3 & \\
\hline 40 & mobile & 3000000 & 0 & 10 & 90000 \\
\hline
\end{tabular}

Процесс моделирования задачи состоял из следующих этапов:

Этап 1. Составить общий список объектов (загрузка карты из файла). Даны три типа объектов: simple - обычный объект, lkks локальная контрольно-корректирующая станция (ЛККС), mobile - мобильный объект. Заданы три характеристики для каждого пункта: $X_{i} ; Y_{i}$; $B$, где $B$ - важность объекта, а также координаты мобильных объектов $X_{m}$, $Y_{m}, B$, доступных для наблюдения в заданное время $t$.

Этап 2. Разбить общий список объектов на два списка

2.1. Составить список мобильных объектов и отсортировать его по времени посещения;

2.2. Составить список не посещённых объектов: обычных пунктов и ЛККС.

Этап 3. Пройти по списку мобильных объектов. Выбрать первый МО и присвоить ему статус текущего. Для каждого мобильного объекта выполнить этап 4. 
Многокритериальная оценка эффективности группового полета летательных аппаратов...

Этап 4. Проверить, остались ли непосещённые объекты в окрестностях текущего мобильного объекта. Если не осталось ни одного объекта (все объекты посещены), перейти к следующему мобильному объекту. Если остались объекты, то найти потенциальный объект по выбранному критерию. После чего вычислить расстояния от текущего объекта (в котором сейчас находится БЛА) до каждого объекта из списка непосещённых. Затем найти минимальное из этих расстояний $R_{\min }$.

Этап 5. Для каждого объекта из списка вычислить значение выбранного критерия из двух представленных: мультипликативного и аддитивного. Для того, чтобы вычислить значение критерия, сначала необходимо вычислить расстояние $R$ от текущего объекта до непосещенного и важность $B$ непосещенного объекта в зависимости от выбранного критерия, значения которых вычисляется по следующим формулам:

Аддитивный критерий:

$$
\sum=(1+0,5)+\frac{R}{R_{\min }} \cdot 0,4+\frac{B}{B_{\max }} \cdot 0,1,
$$

Мультипликативный критерий:

$$
\Pi_{1}=\left(\frac{B}{B_{\max }}+0,5\right)\left(\frac{R}{R_{\min }}+0,4\right)(1+0,1) .
$$

Этап 6. Перейти к следующему мобильному объекту и добавить этот объект в маршрут.
Результаты моделирования маршрута по мультипликативному критерию представлены на рис. 2.

Характеристики этого маршрута таковы:

вес важности $m_{1}: 0,5$;

вес расстояния $m_{2}: 0,4$;

вес времени необслуживания $m_{3}: 0,1$;

суммарная важность: 183.

Результаты моделирования маршрута по аддитивному критерию представлены на рис. 3.

Характеристики второго маршрута таковы: вес важности $m_{1}: 0,1$;

вес расстояния $m_{2}: 0,4$;

вес времени необслуживания $m_{3}: 0,5$;

суммарная важность: 155 .

Оказалось, что суммарная важность обслуженных объектов в маршруте, найденном с помощью мультипликативного критерия, на 18 \% больше важности маршрута, найденного с использованием аддитивного критерия. Исходя из полученных результатов, можно сделать вывод: мультипликативный критерий оказался наиболее удачным для построения маршрута БЛА.

\section{ЗАКЛЮЧЕНИЕ}

Для этапа предполетного планирования маршрутного полета разработан единый критерий оценки эффективности в мультиплика-

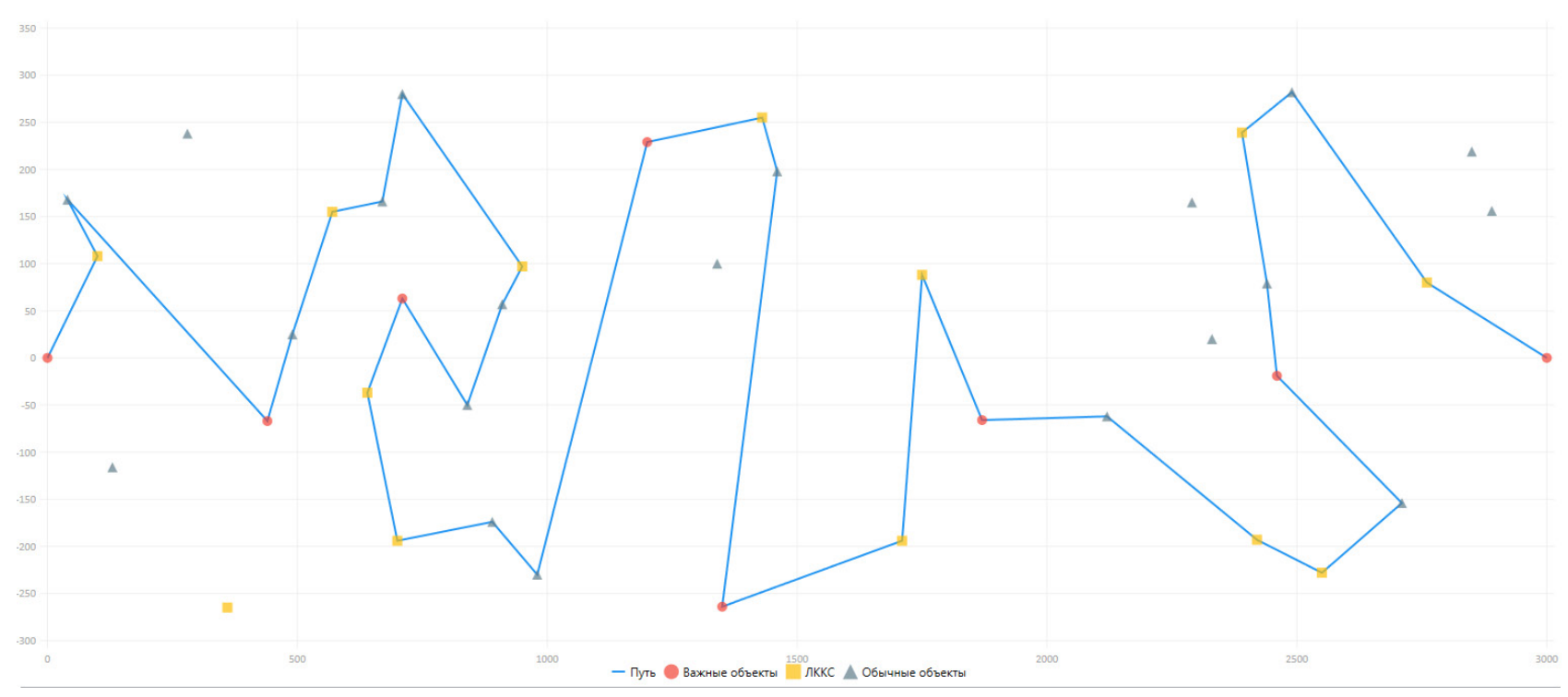

Рис. 2. Результаты компьютерного моделирования марирута с использованием мультипликативного критерия

[Fig.2. Results of computer simulation of the route using a multiplier criterion] 


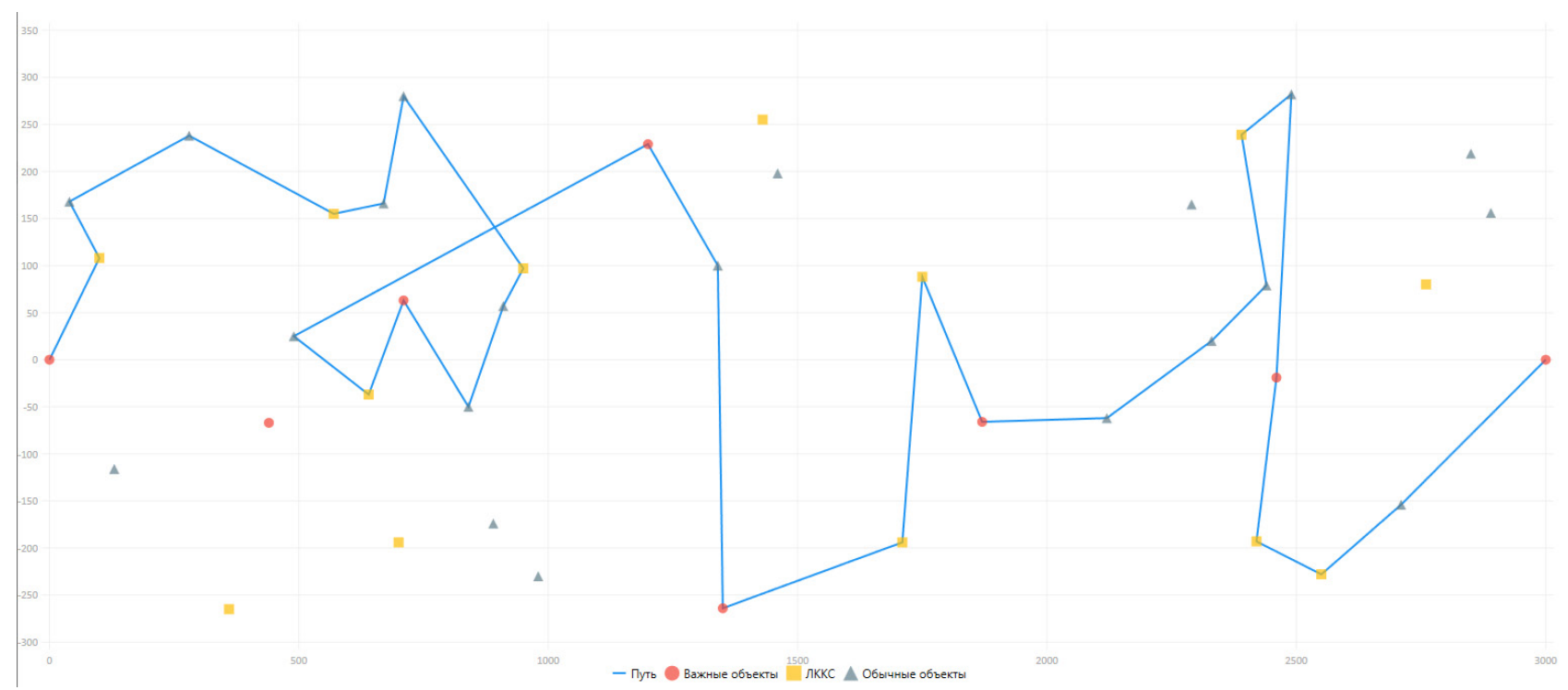

Рис.3. Результаты компьютерного моделирования нахождения маршрута по аддитивному критерию

[Fig.3. Results of computer simulation of route finding by additive criterion]

тивной форме, учитывающий ряд влияющих факторов в многокритериальной задаче. Достоинство предлагаемой нелинейной свертки состоит в том, что для нее достаточно задать ряд весовых коэффициентов, количество которых равно числу этих факторов. Сравнение использования аддитивного и мультипликативного критериев путем компьютерного моделирования показало преимущества мультипликативной формы, при снижении цены разведки наземных объектов примерно на $15 \%$.

\section{КОНФЛИКТ ИНТЕРЕСОВ}

Авторы декларируют отсутствие явных и потенциальных конфликтов интересов, связанных с публикацией настоящей статьи.

Работа выполнена при материальной поддержке грантов РФФИ №17-29-03185 оби_м., № 20-08-00652 $a$.

\section{СПИСОК ЛИТЕРАТУРЫ}

1. Соболь, И. М. Выбор оптимальных параметров в задачах со многими критериями / И. М. Соболь, Р. Б. Статников. - Москва: Наука, 1981. - 110 с.

2. Лебедев, Г. Н. Применение динамического программирования для маршрутизации облета неподвижных пунктов при мониторинге наземной обстановки в контролируемом регионе / Г. Н. Лебедев, А. В. Ефимов // Мехатроника, автоматизация, управление. 2012. - № 1. - C 63-70.

3. Baker, F. B. Item Response Theory: Parameter Estimation Techniques / F. B. Baker, S. H. Kim. - NY: CRC Press, 2004. 528 p.

4. Kang, T. IRT Model Selection Methods for Dichotomous Items / T. Kang, A. S. Cohen // Applies Psychological Measurement. - 2007. - V. 31, N 4. - P. 331-358. doi:10.1177//0146621606299. 233.

5. Chalmers, R. P. Mirt: A Multidimensional Item Response Theory Package for the R Environment / R. P. Chalmers // Journal of Statistical Software. - 2012. V. 48. - N 6. - P. 1-29. DOI: 10.18637/jss.v048.i06

6. Claeskens, G. Model Selection and Model Averaging / G. Claeskens, N. L. Hjort. - NY: Cambridge University Press, 2008. - 332 p. 
Многокритериальная оценка эффективности группового полета летательных аппаратов...

7. Ayala, R. J. The Theory and Practice of Item Response Theory / R. J. Ayala. - NY: Guilford Press, 2009. - 448 p. DOI: 10.1007/s11336-0109179-Z

8. Лебедев, Г. Н. Проблемы построения компьютерных систем обучения специалистов ракетно-космических комплексов / Г. Н. Лебедев, О. В. Романов, А. Ю. Алексеев // Авиакосмическое приборостроение. - 2003. № 9. - С. 25-31.

9. Подготовка операторов безотказного управления сложными подвижными объектами при регулируемом регламенте проведения проверок / С. В. Канушкин [и др.] // Мехатроника, автоматизация, управление. 2012. - № 3. - С. 67 - 70.

10. Шукшунов, В. Е. Тренажерные комплексы и тренажеры. Технологии разработки и опыт эксплуатации / В. Е. Шукшунов, В. В. Циблиев, С. И. Потоцкий. - Москва: Машиностроение, 2005. - 384 с.

11. Кузнецова, Т. И. Постановка задачи оптимального выбора времени при самостоятельном изучении разделов иностранного языка в компьютерном классе с учетом неодинаковой сложности заданий и индивидуальных особенностей обучаемых лиц в студенческой группе / Т. И. Кузнецова, М. Г. Царегородцева // Современные технологии в задачах управления, автоматики и обработки информации: сб. тр. XXV Международной научно-технической конференции. - Алушта, 2016. - C. 209.

12. Лебедев, Г. Н. Применение динамического программирования при автоматизированном обучении операторов управления воздушным движением / Г. Н. Лебедев, С. Д. Ву // Труды МАИ. - 2011. - № 44. - 6 с.

13. Петрушин, В. А. Интеллектуальные обучающие системы / В. А. Петрушин // Известия АН. Техническая кибернетика. - 1993. № 2. - С. 164-190.

14. Лебедев, Г. Н. Решение задачи динамического программирования при безопасном попутном движении воздушных судов / Г. Н. Лебедев, П. Ч. Тин, В. Т. Чан // Труды МАИ. 2012. - № 54. - 7 c.
15. Лебедев, Г. Н. Оценка вектора состояния беспилотного летательного аппарата при наличии в объекте управления нелинейных элементов / Г. Н. Лебедев, А. В. Ефимов Д. А. Михайлин // Вестник Московского авиационного института. - 2012. - № 1. - С. 12 16.

16. Оптимальное управление и контроль безопасности поперечного движения воздушных и речных судов при пересечении их маршрутов / Г. Н. Лебедев [и др.] // Мехатроника, автоматизация, управление. - 2012. № 12. - С. 50-53.

17. Беллман, Р. Динамическое программирование / Р. Беллман. - Москва : Издательство иностранной литературы. - 1960. - 161 с.

18. Зайцев, А. В. Многокритериальность оценки качества операторного управления робототехническими комплексами. Сборник трудов участников VII ВНПК «Современное непрерывное образование и инновационное развитие» / А. В. Зайцев, С. В. Канушкин // Под ред. А. Н. Царькова и И. А. Бугакова. Серпухов: МОУ «ИИФ». - 2017. - С. 841-844.

19. Кузнецова, Т. И. Комплексная оценка качества изучения иностранного языка в виде суммы аддитивной и мультипликативной сверток отдельных показателей освоения различных разделов на всех этапах обучения / Т. И. Кузнецова, М. Г. Царегородцева // Современные технологии в задачах управления, автоматики и обработки информации: сб. тр. XXV Международной научно-технической конференции. - Алушта, 2016. - С. 208.

20. Выбор множества приоритетных наземных объектов наблюдения с помощью беспилотных летательных аппаратов и маршрутизация их полета / В. И. Гончаренко [и др.] // Вестник компьютерных и информационных технологий. - 2019. - № 2. - С. 3-12. DOI: 10.14489 / vkit.2019.02.PP. 003-012

21. Квазиоптимальное распределение авионики на основе клеточного автомата и муравьиного алгоритма / А. В. Кузнецов [и др.] // Вестник Воронеж. гос. ун-та. Сер. Системный анализ и информационные технологии. 2017. - № 4. - С. $38-45$. 
Лебедев Георгий Николаевич - д-р. техн. наук, проф., профессор кафедры «Системы автоматического и интеллектуального управления» Московского авиационного института (национального исследовательского университета).

E-mail: kaf301@mai.ru

ORCID ID: https://orcid.org/0000-0002-1385-0181

Михайлин Денис Александрович - канд. техн. наук, доцент, доцент кафедры «Системы автоматического и интеллектуального управления» Московского авиационного института (национального исследовательского университета).

E-mail: tau_301@mail.ru

ORCID ID: https://orcid.org/0000-0002-8822-9138

Царева Ольга Юрьевна - магистрант 2-го года обучения кафедры «Системы автоматического и интеллектуального управления» Московского авиационного института (национального исследовательского университета).

E-mail: olyatsareva@yandex.ru

ORCID ID: https://orcid.org/0000-0002-4665-7556

Чернякова Марина Евгеньевна - стажер Московского филиала ООО «Группа-Кронштадт». E-mail: marina.chernyakova@list.ru

ORCID ID: https://orcid.org/0000-0002-7379-9672

DOI: https://doi.org/10.17308/sait.2020.2/2913

Received 07.06.2020

Acceptedb 15.06.2020

ISSN 1995-5499

\title{
MULTI-CRITERIA EVALUATION OF THE EFFICIENCY OF FORMATION FLYING USING THE MULTIPLICATIVE FORM
}

\author{
(C) 2020 G. N. Lebedev ${ }^{1}$, D. A. Mikhailin', O. Y. Tsareva ${ }^{\bowtie 1}$, M. E. Chernyakova² \\ ${ }^{1}$ Moscow Aviation Institute (National Research University) \\ 4, Volokolamskoye Highway, 125993 Moscow, Russian Federation \\ ${ }^{2}$ Kronstadt Group LLC \\ 18, Andropova Avenue, 115342 Moscow, Russian Federation
}

\begin{abstract}
Annotation. The article considers the multi-criteria problem of evaluating the efficiency of formation flying of manned and unmanned aircraft when handling the flow of requests. Using dynamic programming we designed a model for assigning dynamic priorities that assess the importance of the completed requests. A single criterion for assessing the efficiency was selected using the multiplicative form, in which the number of cofactors is equal to the number of factors taken into account. Computer simulation demonstrated that the multiplicative form is more effective for planning than the additive one due to the increase in the total importance of the completed requests during cross-country flight.

Keywords: requests processing, dynamic programming, computer modelling, efficiency criteria.
\end{abstract}

Tsareva Olga Y.

e-mail: olyatsareva@yandex.ru 


\section{CONFLICT OF INTEREST}

The authors declare the absence of obvious and potential conflict of interest related to the publication of this article.

\section{REFERENCES}

1. Sobol I. M., Statnikov R. B. Selection of the optimal parameters in problems with many criteria. Moscow : Science, 1981. 110 p.

2. Lebedev G. N., Efimov A. V. Application of the dynamic programming for the fixed point flight routing at the monitoring of the ground situation in the controlled region. Mechatronics, automation, control. 2012. No 1. P. 63-70.

3. Baker F. B., Kim S. H. Item Response Theory: Parameter Estimation Techniques. NY : CRC Press, 2004. 528 p.

4. Kang T., Cohen A. S. IRT Model Selection Methods for Dichotomous Items. Applies Psychological Measurement. 2007. V.31. N 4. P. 331358. doi:10.1177//0146621606299.233.

5. Chalmers R. P. Mirt: A Multidimensional Item Response Theory Package for the R Environment. Journal of Statistical Software. 2012. V. 48. N 6. P. 1-29. DOI: 10.18637/jss.v048.i06

6. Claeskens G., Hjort N. L. Model Selection and Model Averaging. NY : Cambridge University Press, 2008. 332 p.

7. Ayala R. J. The Theory and Practice of Item Response Theory. NY : Guilford Press, 2009. 448 p. DOI: $10.1007 / \mathrm{s} 11336-010-9179-\mathrm{Z}$

8. Lebedev G. N., Romanov O. V., Alexeev A. Yu. Problems of the computer systems construction for training of the rocket and space systems specialists. Aerospace Instrumentation. 2003. No 9. P. 25-31.

9. Kanushkin S. V. [et al.] Preparation of the operators of the trouble-free control of the complex movable objects at the regulated inspection procedure. Mechatronics, automation, control. 2012. No 3. P. 67-70.

10. Shukshunov V. E., Tsifiev V. V., Pototsky S. I. Gym complexes and simulators. Development technologies and operation experience. Moscow : Mashinostroenie, 2005. 384 p.

11. Kuznetsova T. I., Tsaregorodtseva M. G. Problem setting of the optimal time choice at in- dependent studying of the foreign language sections in a computer class taking into account the uneven complexity of the tasks and individual features of the students in the student group (in Russian). Modern technologies in problems of control, automation and information processing: a collection of articles of XXV International scientific and technical conference. Alushta, 2016. P. 209.

12. Lebedev G. N., Vu S. D. Application of the dynamic programming at the automated training of the air traffic control operators. 2011. No 44. $6 \mathrm{p}$.

13. Petrushin, V. A. Intellectual teaching systems. Izvestia NA. Technical cybernetics. 1993. No 2. P. 164-190.

14. Lebedev G. N., Tin P. Ch., Chan V. T. Solution of the dynamic programming problem at a safe by-product motion of the aircrafts. Proc. of MAI. 2012. No 54.7 p.

15. Lebedev G. N., Efimov A. V., Mikhailin D. A. Estimation of the drone aircraft state vector at presence of the nonlinear elements in the control object. Vestnik of Moscow Aviation Institute. 2012. No 1. - P. 12-16.

16. Lebedev G. N. [et al.] Optimal control and safety control of the aircrafts and river vessels transverse motion at their routes crossing. Mechatronics, automation, control. 2012. No 12. P. 50-53.

17. Bellman R. Dynamic programming. Moscow : Publishing house of foreign literature. 1960. $161 \mathrm{p}$.

18. Zaitsev A. V., Kanushkin S. V. Multi-criteria of quality estimation of operator's control of robotic complexes. Collection of works of the participants of VII VNPK "Modern continuous education and innovative development". Under edition of A. N. Tsarkova and I. A. Bugakov. Serpukhov : MOU “IIF”. 2017. P. 841-844.

19. Kuznetsova T. I., Tsaregorodtseva M. G. Complex quality estimation of foreign language studying in the form of sum of the additive and multiplying convolution of the separate indexes of mastering of the various sections at all stages of training. Modern technologies in problems of management, automatics and information processing: a collection of articles of XXV International scientific and technical conference. 2016. P. 208. 
20. Goncharenko V. I. [et al.] Selection of the set of the priority ground objects of observation with the help of the unmanned air vehicles and routing of their flight. Vestnik of computer and information technologies. 2019. No 2. P. 3-12. DOI: 10.14489 / vkit.2019.02.PP. 003-012
21. Kuznetsov A. V. [et al.] Quasi-optimal distribution of avionics on the basis of the cellular automaton and the ant algorithm. Vestnik Voronezh. str. Ser. System analysis and information technologies. 2017. No 4. P. 38-45.

Lebedev Georgy N. - DSc in Technical Sciences, Professor, Department of Automatic and Intelligent Control Systems, Moscow Aviation Institute (National Research University).

E-mail: kaf301@mai.ru.

ORCID ID: https://orcid.org/0000-0002-1385-0181

Mikhailin Denis A. - PhD in Technical Sciences, Associate Professor, Department of Automatic and Intelligent Control Systems, Moscow Aviation Institute (National Research University).

E-mail: tau_301@mail.ru

ORCID ID: https://orcid.org/0000-0002-8822-9138

Tsareva Olga Y. - graduate student, Department of Automatic and Intelligent Control Systems, Moscow Aviation Institute (National Research University).

E-mail: olyatsareva@yandex.ru

ORCID ID: https://orcid.org/0000-0002-4665-7556

Chernyakova Marina E. - trainee at the Moscow branch of Kronstadt Group LLC.

E-mail: marina.chernyakova@list.ru

ORCID ID: https://orcid.org/0000-0002-7379-9672 